\title{
Expression of neural cell adhesion molecule and polysialic acid in human bone marrow- derived mesenchymal stromal cells
}

\author{
Maria S. Skog ${ }^{1 *}$, Johanna Nystedt ${ }^{2}$, Matti Korhonen², Heidi Anderson ${ }^{1,3}$, Timo A. Lehti ${ }^{1}$, Maria I. Pajunen ${ }^{1,4 \dagger}$ \\ and Jukka Finne ${ }^{1 \dagger}$
}

\begin{abstract}
Background: In order to develop novel clinical applications and to gain insights into possible therapeutic mechanisms, detailed molecular characterization of human bone marrow-derived mesenchymal stromal cells (hBM-MSCs) is needed. Neural cell adhesion molecule (NCAM, CD56) is a transmembrane glycoprotein modulating cell-cell and cell-matrix interactions. An additional post-translational modification of NCAM is the a2,8-linked polysialic acid (polySia). Because of its background, NCAM is often considered a marker of neural lineage commitment. Generally, hBM-MSCs are considered to be devoid of NCAM expression, but more rigorous characterization is needed.
\end{abstract}

Methods: We have studied NCAM and polySia expression in five hBM-MSC lines at mRNA and protein levels. Cell surface localization was confirmed by immunofluorescence staining and expression frequency in the donor-specific lines by flow cytometry. For the detection of poorly immunogenic polySia, a fluorochrome-tagged catalytically defective enzyme was employed.

Results: All five known NCAM isoforms are expressed in these cells at mRNA level and the three main isoforms are present at protein level. Both polysialyltransferases, generally responsible for NCAM polysialylation, are expressed at mRNA level, but only very few cells express polySia at the cell surface.

Conclusions: Our results underline the need for a careful control of methods and conditions in the characterization of MSCs. This study shows that, against the generally held view, clinical-grade hBM-MSCs do express NCAM. In contrast, although both polysialyltransferase genes are transcribed in these cells, very few express polySia at the cell surface. NCAM and polySia represent new candidate molecules for influencing MSC interactions.

Keywords: Bone marrow, Mesenchymal stromal cell, Clinical grade, Neural cell adhesion molecule, Polysialic acid

\section{Background}

Human bone marrow-derived mesenchymal stromal cells (hBM-MSCs) are attractive candidates for cellular therapy, regenerative medicine, and tissue engineering. They are adult progenitor cells that hold potential for fast clonal expansion, secretion of trophic and immunomodulatory factors, and multilineage differentiation. In addition, they are relatively easy to harvest, isolate, and

\footnotetext{
* Correspondence: maria.skog@helsinki.fi

${ }^{\dagger}$ Equal contributors

${ }^{1}$ Biochemistry and Biotechnology, Department of Biosciences, University of Helsinki, P.O. Box 56, Fl-00014 Helsinki, Finland

Full list of author information is available at the end of the article
}

culture. However, it is widely acknowledged that following in-vitro culture, MSCs undergo replicative senescence and appear to lose their beneficial traits [1]. Furthermore, MSC cultures typically consist of a heterogeneous mixture of cells at different stages of commitment and potential $[1,2]$. In order to develop novel clinical applications and to gain more insight into the possible therapeutic mechanisms, detailed molecular characterization of hBM-MSCs is needed. This will ultimately improve the safety and efficacy of cell therapy.

Neural cell adhesion molecule (NCAM, CD56) is a calcium-independent binding protein engaged in homophilic cell-cell and heterophilic cell-matrix interactions [3]. 
NCAM is transcribed from a single gene, but exists in multiple isoforms as a result of alternative splicing [4]. In humans, at least five distinct NCAM isoforms are known. A modification of NCAM results posttranslationally from the addition of linear polymers of $\mathrm{N}$-acetylneuraminic acid units, polysialic acid (polySia) $[5,6]$. Being a large negatively charged and highly hydrated structure, polySia regulates NCAM activity by altering its biophysical properties [7]. PolySia has an important role in maintaining developmental plasticity and cell migration in tissues by influencing cellular interactions [8]. In addition, it is involved in various medical conditions such as tissue repair, neurodegenerative diseases, and progression of metastatic cancers $[9,10]$.

Expression of NCAM and polySia is strong and dynamic during embryogenesis, decreases and focuses during development, and is limited to just few tissues and cell types in the adult $[11,12]$. Because of its original discovery site, NCAM is often considered a marker of neural lineage commitment $[13,14]$. However, it is known that expression of NCAM and polySia is widespread during organogenesis, particularly in undifferentiated mesenchymal cells [15]. Many in-vivo and in-vitro studies in animal models suggest that NCAM is an important regulator of cell migration and condensation during skeletal development [16-18]. Hence, NCAM and polySia represent promising candidates for influencing MSC interactions.

It is generally held that hBM-MSCs do not express NCAM [19-25], while, for example, placental and umbilical cord blood-derived MSCs are eminently NCAM positive [21, 24, 25]. Brooke et al. [26] have reported that hBM-MSCs do express NCAM at the mRNA level, but protein expression was not investigated. NCAM protein expression, which may indicate increased chondrogenic potential, has been reported in a small fraction of primary bone marrow mononuclear cells $(0.5-5.5 \%)$, but expression diminished over time in culture [27, 28]. In contrast, murine BM-MSCs predominantly express NCAM, which plays a crucial role, for example, in hematopoiesis [29]. Furthermore, experiments with NCAM knockout mice have shown reduced multilineage differentiation potential of BM-MSCs compared with wild-type controls $[30,31]$. Thus, because of the role of NCAM and polySia in the control of cellular differentiation and interaction, it is important to reliably determine whether they are expressed in clinical-grade hBM-MSCs.

In this study, we have investigated the expression status of NCAM and polySia in clinical-grade hBM-MSCs using a variety of methods. We have concentrated particularly on NCAM expression, because we observed a striking discrepancy between our findings and previous reports [19-25]. Furthermore, NCAM is the most studied molecule of the immunoglobulin superfamily of cell adhesion molecules (CAMs), but has been largely neglected in stem cell research despite its role as a developmental regulator. This study clearly demonstrates the need for comprehensive analyses and careful control of methods in the characterization of MSCs. Gene and protein expression analyses show that these cells do, in fact, express NCAM. In contrast, although polysialyltransferases are transcribed in these cells, very few express polySia on the cell surface.

\section{Methods \\ Cells}

The culture protocol developed by Laitinen et al. [32] for clinical-grade MSCs based on platelet lysate was utilized in this study. Bone marrow was collected from five healthy volunteer donors (donor 067: female, age 24; donor 068: female, age 31; donor 069: female, age 30; donor 072: female, age 21; donor 073: female, age 21). Bone marrow was aspirated under local anesthesia from the posterior iliac crest and collected in heparinized tubes after signed informed consent according to the Declaration of Helsinki. The protocol was approved by the ethics committee of the Hospital District of Helsinki and Uusimaa (Finland). The isolation and characterization of hBM-MSCs has been described in detail previously [32]. The isolated cells were cultured in heparinized (LEO Pharma, Ballerup, Denmark) low-glucose Dulbecco's modified Eagle's medium (DMEM; Gibco, Life Technologies, Paisley, UK), supplemented with $10 \%$ platelet lysate (Finnish Red Cross Blood Service, Helsinki, Finland), and $100 \mathrm{U} / \mathrm{ml}$ penicillin and $100 \mu \mathrm{g} / \mathrm{ml}$ streptomycin (Gibco) according to Laitinen et al. [32]. The medium was changed twice weekly and the cultures were passaged when subconfluent (80\% confluency) and subcultured at 10001500 cells $/ \mathrm{cm}^{2}$. The hBM-MSCs used in this study were freshly analyzed (i.e., noncryopreserved) at passage 2 or 3 .

Human neuroblastoma SK-N-SH cells (ATCC, Manassas, VA, USA) were cultured in high-glucose DMEM (Sigma, St. Louis, MO, USA), supplemented with $10 \%$ fetal bovine serum (FBS) (HyClone; Thermo Scientific, Logan, UT, USA), and $100 \mathrm{U} / \mathrm{ml}$ penicillin and $100 \mu \mathrm{g} / \mathrm{ml}$ streptomycin (Gibco). Inactive endosialidase-GFP fusion protein developed by Jokilammi et al. [33] and magnetic GFP-Trap-M beads (Chromotek, Planegg-Martinsried, Germany) were employed to fractionate the strongly polySia-expressing cell population (kSK-N-SH) to be used as a positive control. First, cells were labeled with inactive endosialidase-GFP fusion protein in phosphate-buffered saline (PBS; containing $1.06 \mathrm{mM}$ potassium phosphate monobasic, $155.2 \mathrm{mM}$ sodium chloride, and $2.97 \mathrm{mM}$ sodium phosphate dibasic) for 1 hour on ice. Labeled cells were then mixed with GFP-Trap ${ }^{\circ}-\mathrm{M}$ beads and separated magnetically until the bead-associated cells were perceptibly gathered to the proximity of the magnet. Supernatant 
was discarded and isolated cells were washed with PBS. Washing and magnetic separation was repeated 10 times. Lastly, the isolated cells were plated on a cell culture dish and cultured accordingly. The NCAM and polySia expression status was analyzed with flow cytometry; the proportion of NCAM and polySia-expressing cells was $98.3 \%$.

All cells were cultured under a humidified atmosphere at $37{ }^{\circ} \mathrm{C}$ and with $5 \% \mathrm{CO}_{2}$.

\section{Differentiation of hBM-MSCs}

Adipogenic differentiation of hBM-MSCs was performed as described previously by Laitinen et al. [32]. The cells were cultured in adipogenic differentiation condition for 1-2 weeks. After differentiation the cells were fixed with $4 \%$ paraformaldehyde in PBS for Sudan III staining.

Osteogenic differentiation of hBM-MSCs was carried out essentially as described previously by Laitinen et al. [32]. The cells were cultured in osteogenic medium for 3-4 weeks. After differentiation the cells were fixed with $4 \%$ paraformaldehyde in PBS for von Kossa staining.

Chondrogenic differentiation was performed as described previously by Skog et al. [34]. The pellet cultures were maintained up to 4 weeks, changing the medium twice a week. After differentiation the cell pellets were fixed with $10 \%$ formalin, embedded in paraffin, and cut into $5 \mu \mathrm{m}$ sections for Safranin O staining.

\section{Qualitative reverse transcription PCR}

NCAM isoform-specific sequences were obtained from NCBI Unigene (http://www.ncbi.nlm.nih.gov/unigene). Primers were designed using NCBI Primer Blast (http:// www.ncbi.nlm.nih.gov/tools/primer-blast) and optimized by OligoEvaluator ${ }^{\mathrm{mew}}$ (http://www.sigmaaldrich.com/technicaldocuments/articles/biology/oligo-evaluator.html) or Tm Calculator (https://www.thermofisher.com/fi/en/home/ brands/thermo-scientific/molecular-biology/molecularbiology-learning-center/molecular-biology-resource-library/ thermo-scientific-web-tools/tm-calculator.html) PCR web tools. All commercial kits were used according to the manufacturer's instructions. Total RNA was isolated using the High Pure RNA Isolation Kit (Roche Diagnostics, Mannheim, Germany). Reverse transcription was performed with High Capacity RNA-to-cDNA Kit (Applied Biosystems, Foster City, CA, USA) with $1 \mu \mathrm{g}$ of total RNA. A control reaction omitting the reverse transcriptase was prepared for each sample. PCRs were performed in $20 \mu \mathrm{l}$ final volume with Phusion Hot Start II High-Fidelity DNA Polymerase (Thermo Scientific, Waltham, MA, USA) for all NCAM isoforms, polysialyltransferases (ST8SIA2 and ST8SIA4), and controls, except that OneTaq Hot Start DNA Polymerase (New England BioLabs, Ipswich, MA, USA) was used for isoform NCAM-120.

Human NCAM isoform and polysialyltransferasespecific PCR primers were used for the amplification (Table 1). For Phusion Hot Start II High-Fidelity DNA Polymerase, the reaction mixture was composed of 0.02 $\mathrm{U} / \mu \mathrm{l}$ of DNA polymerase, Phusion HF Buffer (with $1.5 \mathrm{mM} \mathrm{MgCl}_{2}$ ), $0.2 \mathrm{mM}$ dNTPs, $0.5 \mu \mathrm{M}$ primers, $3 \%$ DMSO, sterile water (Baxter Healthcare, Zürich, Switzerland), and $2.0 \mu \mathrm{l}$ of template. For OneTaq Hot Start DNA Polymerase, the reaction mixture was composed of $0.025 \mathrm{U} / \mu \mathrm{l}$ of DNA polymerase, OneTaq Standard Reaction Buffer (with $1.8 \mathrm{mM} \mathrm{MgCl}_{2}$ ), $0.2 \mathrm{mM}$

Table 1 Primers used in the study

\begin{tabular}{|c|c|c|c|c|}
\hline Primer & UniGene & Forward $\left(5^{\prime} \rightarrow 3^{\prime}\right)$ & Reverse $\left(5^{\prime} \rightarrow 3^{\prime}\right)$ & $\begin{array}{l}\text { Amplicon size } \\
\text { (base pairs) }\end{array}$ \\
\hline GAPDH & Hs.544577 & GAAGGTGAAGGTCGGAGTC & GAAGATGGTGATGGGATTTC & 225 \\
\hline NCAM-All & Hs.503878 & GGACTTCTACCCGGAACATCAG & CGAGCTTAGGTGCACTGGG & \\
\hline $\begin{array}{l}\text { NCAM-140 (Variant 1), } \\
\text { NCAM-125 (Variant 4) }\end{array}$ & & & & 798 \\
\hline NCAM-180 (Variant 2) & & & & 828 \\
\hline NCAM-120 (Variant 3) & & & & 903 \\
\hline NCAM Variant 5 & & & & 906 \\
\hline NCAM-180 & NM_181351.4 & GCTTCGTGGACTCGACCAG & GCAGATGTACTCTCCGGCATC & 126 \\
\hline NCAM-140 & NM_000615.6 & CGAAGAAAAGACTCTGGATGG & TCATGCTITGCTCTCGTTCT & 1498 \\
\hline NCAM-125/140 & NM_001242608.1 / NM_000615.6 & CGAAGAAAAGACTCTGGATGG & GTTCCCCTTGGACTGGC & 755 \\
\hline NCAM-125 & NM_001242608.1 & CAGCCAGTCCAAGGGG & TGTAGGATGCAGAATTGCCTC & 346 \\
\hline NCAM120/125 & NM_001076682.3 / NM_001242608.1 & GAGTATGAGGTCTACGTGGTGGC & GCAGAGAAAAGCAATGAGACAAAG & 152 \\
\hline NCAM-120 & NM_001076682.3 & TCTGCTAGCTCGTCTACCCC & CCAAAGGGGGCACTGATCTT & 541 \\
\hline NCAM Variant 5 & NM_001242607.1 & GCTTCGTGGACTCGACCAG & GCAGATGTACTCTCCGGCATC & 204 \\
\hline ST8SIA2 & Hs.302341 & GGGCAACTCGGGGGTCTTGC & AAGGCCCGCTGGATGACCGA & 162 \\
\hline ST8SIA4 & Hs.308628 & ACAGGCGCCGGACACTAAACA & TGCAGCAAACTCCACCACAGGA & 200 \\
\hline
\end{tabular}


dNTPs, $0.2 \mu \mathrm{M}$ primers, $1.5 \mathrm{M}$ betaine (Sigma), sterile water (Baxter Healthcare), and $2 \mu \mathrm{l}$ of template. Amplification was performed for 35 cycles. The PCR products were analyzed by agarose gel electrophoresis. Glyceraldehyde 3-phosphate dehydrogenase $(G A P D H)$ was used as the reference gene.

\section{Western blot}

Whole-cell extracts were prepared by lysing the cells in radioimmunoprecipitation assay (RIPA) buffer $\mathrm{pH} 6.8$ containing $50 \mathrm{mM}$ Trizma ${ }^{\circ}$ base, $150 \mathrm{mM}$ sodium chloride, $0.5 \%$ sodium deoxycholate (all from Sigma), and $1 \%$ Triton X-100 (Roche Life Sciences, Indianapolis, IN, USA), supplemented with EDTA-free protease inhibitor cocktail (Thermo Scientific, Rockford, IL, USA). For removal of polySia, $10 \mu \mathrm{g} / \mathrm{ml}$ of active endosialidase-GFP fusion protein [35] was added and the samples were incubated at $37{ }^{\circ} \mathrm{C}$ for 45 minutes. Samples were mixed with Laemmli sample buffer, loaded, and run on 4-20\% Mini-PROTEAN ${ }^{\circ}$ TGX $^{\text {ma }}$ Precast gradient gel (Bio-Rad Laboratories, Hercules, CA, USA) or 7 \% SDS-polyacrylamide gel. Proteins were electrotransferred from the gels to nitrocellulose membranes (GE Healthcare, Little Chalfont, UK) overnight at $4{ }^{\circ} \mathrm{C}$. The membrane was blocked with $3 \%$ skimmed milk powder in PBS with $0.1 \%$ Tween- 20 for 3 hours at $4{ }^{\circ} \mathrm{C}$.

For NCAM detection, the membrane was incubated with a primary antibody mixture containing $0.33 \mu \mathrm{g} / \mathrm{ml}$ rabbit polyclonal anti-human NCAM antibody (AB5032; Millipore, Temecula, CA, USA) and $0.067 \mu \mathrm{g} / \mathrm{ml}$ mouse monoclonal anti-human NCAM antibody (123C3; Santa Cruz, Dallas, Texas, USA) in blocking solution overnight at $4{ }^{\circ} \mathrm{C}$. Because anti-NCAM antibodies may detect different isoforms with varying affinity in western blot, two primary antibodies were used simultaneously to ensure accurate detection of all isoforms [36]. After three washes, the membrane was incubated with a secondary antibody mixture containing HRP-conjugated anti-rabbit secondary antibody 1:3000 and anti-mouse secondary antibody 1:3000 (both from Cell Signaling, Danvers, MA, USA) in blocking solution for 2 hours at room temperature. The immunoblots were developed with SuperSignal $^{\circ}$ West Pico Chemiluminescent Substrate (Thermo Scientific). Secondary antibody control confirmed the specificity of the labeling, and $\alpha$-Tubulin (B51-2; Sigma) served as a loading control.

\section{Flow cytometry}

Cells were double labeled with $10 \mu \mathrm{g} / \mathrm{ml}$ of rabbit polyclonal anti-human NCAM antibody (AB5032; Millipore) followed by AlexaFluor 647-labeled goat anti-rabbit secondary antibody (Molecular Probes, Invitrogen, Eugene, OR, USA) and inactive endosialidase-GFP fusion protein [33] in PBS. Parallel samples were labeled with
AlexaFluor 647 mouse anti-human alkaline phosphatase antibody (B4-78; BD Biosciences, San Jose, CA, USA) and FITC mouse anti-human CD44 antibody (BD Biosciences) according to the manufacturer's instructions. Appropriate fluorescence minus one (FMO) controls were used for analysis. The cells were analyzed with FACS LSR II flow cytometer and FACSDiva 5.0.3 software (BD Biosciences). Cell debris and dead cells were excluded from the analysis based on physical parameters and propidium iodide (PI) fluorescence probing for cell viability (proportion of positive cells $1.2-7.0 \%$, data not shown).

\section{Immunocytochemistry}

For immunocytochemical staining, cells were grown on glass coverslips and fixed with $4 \%$ paraformaldehyde in PBS. Nonspecific binding was blocked with $1.5 \%$ normal horse serum (Vector Laboratories, Burlingame, CA, USA) in PBS. Cells were labeled with $10 \mu \mathrm{g} / \mathrm{ml}$ of rabbit polyclonal anti-human NCAM antibody (AB5032; Millipore) followed by AlexaFluor 647 conjugated goat anti-rabbit secondary antibody (Molecular Probes) and inactive endosialidase-GFP fusion protein [33], all in PBS. Cover slips were mounted with ProLong Mounting Medium with DAPI (Molecular Probes). The staining was visualized with an Olympus BX50F-3 microscope and imaged by a PCO CCD Imaging SensiCam color camera and Image-Pro Plus 4.0 software.

\section{Results}

\section{Multilineage differentiation of hBM-MSCs}

The multilineage differentiation assay was performed to verify the differentiation capacity of hBM-MSCs according to the International Society for Cellular Therapy (ISCT) minimal criteria for MSCs [37]. All hBM-MSC lines displayed typical MSC differentiation capacity along the adipogenic, osteogenic, and chondrogenic lineages (Fig. 1).

\section{Expression of NCAM and polysialyltransferase mRNA in hBM-MSCs}

To gain an overview of NCAM mRNA expression, qualitative reverse transcription PCR analysis was performed with isoform-specific primer pairs (Fig. 2a). The reverse transcription PCR analysis revealed distinct expression of all NCAM isoform mRNAs in the three analyzed hBM-MSC lines (Fig. 2b).

NCAM polysialylation is catalyzed by two Golgi resident enzymes, polysialyltransferases ST8SIA2 and ST8SIA4 (Fig. 3a). Transcription of polysialyltransferases was analyzed to confirm the prospect of NCAM polysialylation. The analysis shows unambiguously that both transferase mRNAs are transcribed in the three hBM-MSC lines analyzed (Fig. 3b). 


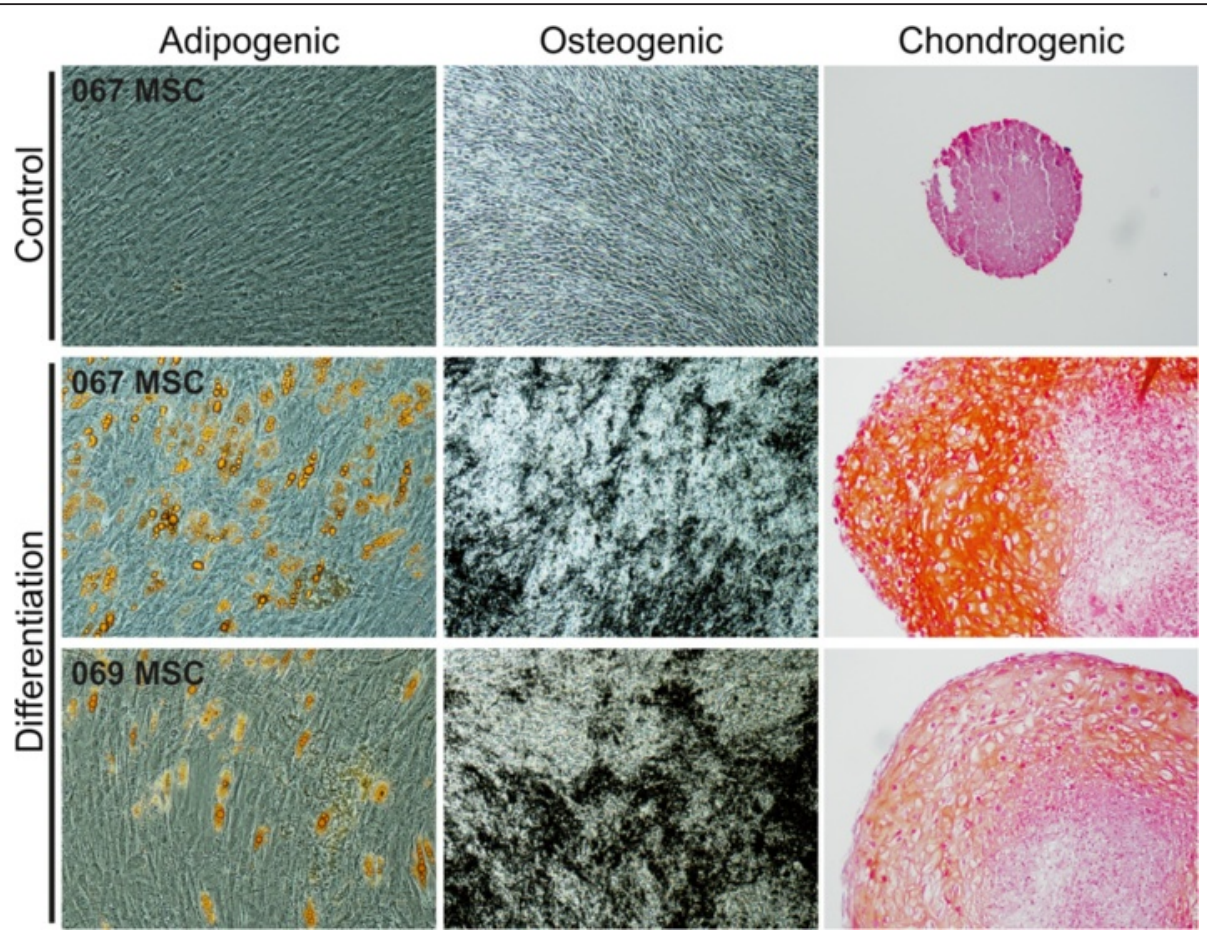

Fig. 1 Multilineage differentiation of hBM-MSCs. Representative light microscopic photographs of control and differentiated hBM-MSCs. Adipogenic differentiation was confirmed by Sudan III staining, osteogenic differentiation by von Kossa staining, and chondrogenic differentiation by Safranin $\mathrm{O}$ staining. All hBM-MSC lines displayed typical differentiation capacity. Adipogenic and chondrogenic differentiation images were taken at 10x lens objective magnification, osteogenic differentiation images at $4 \times$ lens objective magnification. MSC mesenchymal stromal cell

\section{Surface expression of NCAM and polySia in hBM-MSCs}

Using commercially available polyclonal human-specific NCAM antibody, we detected cell surface NCAM expression on hBM-MSCs in flow cytometry (Fig. 4a), indicating that NCAM mRNA is translated into protein. All five hBM-MSC lines included in this study expressed NCAM on the cell surface. However, its occurrence was heterogeneous, because not all cells in the populations expressed NCAM at detectable levels. Differences between donor-specific hBM-MSC lines were also observed, the proportion of positive cells ranging from $23.6 \pm 0.8 \%$ to $88.5 \pm 7.4 \%$ (Fig. 4 b). Concurrent polySia detection was performed with inactive endosialidaseGFP fusion protein that binds to polySia. Surprisingly, only very few hBM-MSCs expressed polySia (Fig. 4a), the proportion of positive cells ranging from $0.5 \pm 0.2 \%$ to $4.4 \%$ (Fig. 4b). All polySia-expressing cells were simultaneously labeled positively for NCAM, the main carrier of polySia.

For comparison, cell surface expression of CD44 and tissue nonspecific alkaline phosphatase (TNAP) was also analyzed from hBM-MSC lines. Flow cytometry data indicate that CD44, a common MSC marker, is fully expressed in all of the lines (>99\%, Fig. 4b). On the other hand, expression of the early osteogenic marker TNAP was more heterogeneous, ranging from $55.3 \%$ to
$92.8 \pm 3.7 \%$ (Fig. 4b). No correlation was found between NCAM and TNAP expression.

\section{Expression of NCAM isoforms in hBM-MSCs}

NCAM protein expression was further confirmed by western blot analysis (Fig. 5). Bands of approximately 180,140 , and $120 \mathrm{kDa}$ in size were detected in all hBMMSC lines analyzed, indicating the presence of the three main isoforms of NCAM. At the protein level, hBMMSCs seem to express predominantly NCAM-180 and other isoforms to a lesser extent. However, distinction between NCAM-125 and NCAM-120 was not possible because the bands overlap. Some variation in the expression of different isoforms was observed between the hBM-MSC lines, MSC 069 expressing different isoforms in more equal manner compared with other hBM-MSC lines. Because polySia may affect NCAM mobility in gel electrophoresis, endosialidase [35] was used to treat a set of parallel samples. In agreement with high polySia expression on kSK-N-SH cells (Fig. 5), endosialidase treatment revealed the presence of NCAM in these cells. In contrast, endosialidase treatment did not uncover additional NCAM in hBM-MSCs. Altogether, the western blot results demonstrate that hBM-MSCs express various NCAM isoforms also at the protein level. 


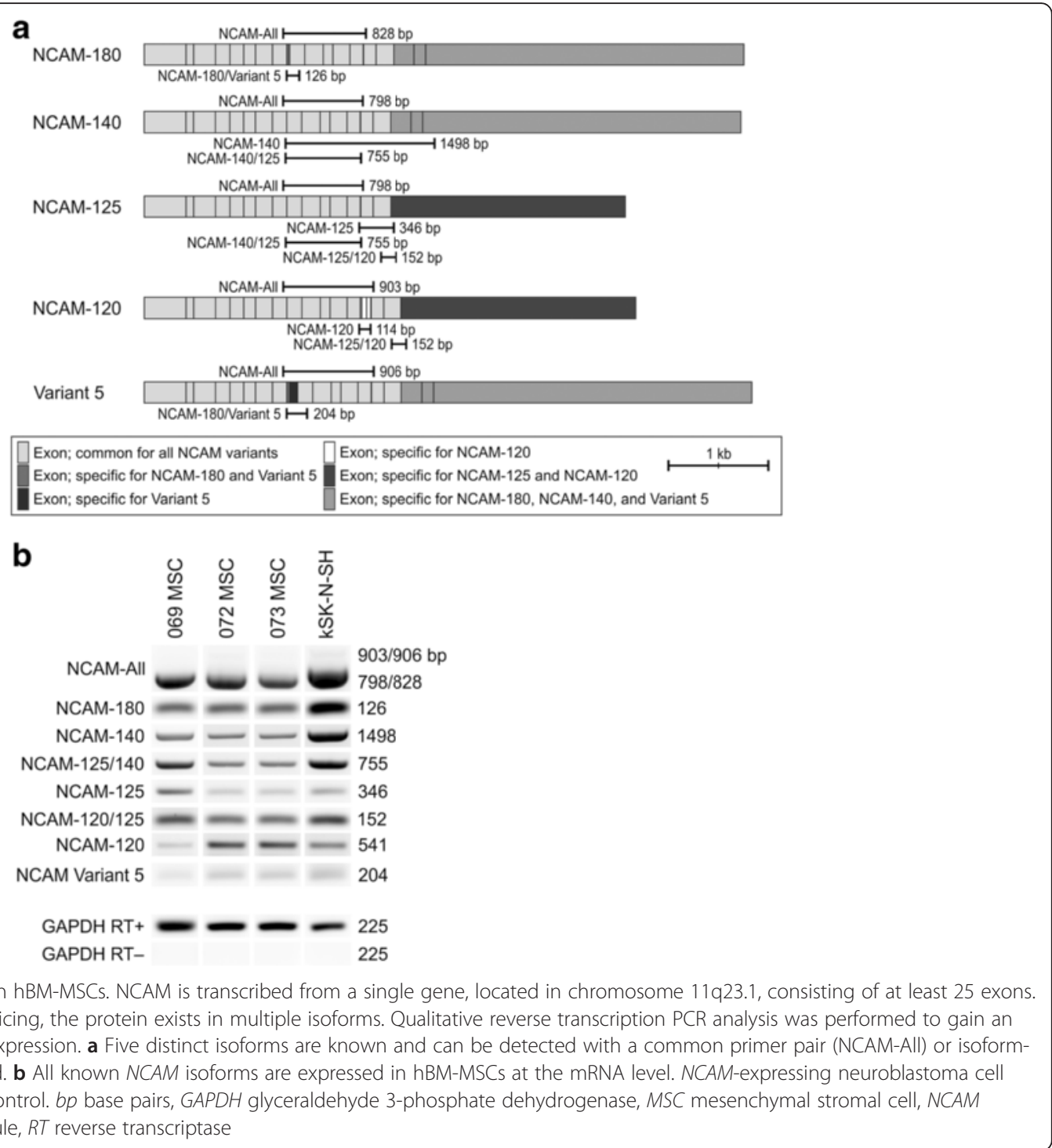

\section{Cellular localization of NCAM and polySia}

To determine the subcellular localization of NCAM and polySia, immunocytochemical detection was utilized. NCAM appears to be expressed in a clustered manner over the cell surface in hBM-MSCs (Fig. 6). In contrast, NCAM expression was even and smooth on the reference neuroblastoma cell line kSK-N-SH. NCAM expression in kSK-N-SH seems to be more intense around cell-cell contact sites, whereas this was not the case for hBM-MSCs.

Compared with NCAM, very little polySia expression was detected in hBM-MSCs (Fig. 7). Grainy polySia expression was detected mainly on cellular extensions. This was very different from the polySia expression in kSK-N-SH cells, where polySia is distributed smoothly all over the cell surface. In kSK-N-SH cells, polySia expression, together with NCAM, appears to be more intense in cell-cell contacts; however, in hBM-MSCs such condensation was not detected. Altogether, the immunocytochemistry results confirm that NCAM protein is expressed on the cell surface.

\section{Discussion}

Previous studies have shown that NCAM is a modulator of various cellular functions such as cell-cell and cellmatrix interactions, neuronal migration, neurite outgrowth, and synapse formation [3, 38, 39]. Furthermore, it is an important regulator of cell migration and condensation during skeletal development [16-18]. It is noteworthy that NCAM is not merely an adhesion molecule, but initiates the formation of intracellular membrane-proximal signaling complexes, thereby activating a complex network of signal transduction [40]. In addition, polySia affects cellular functions such as 


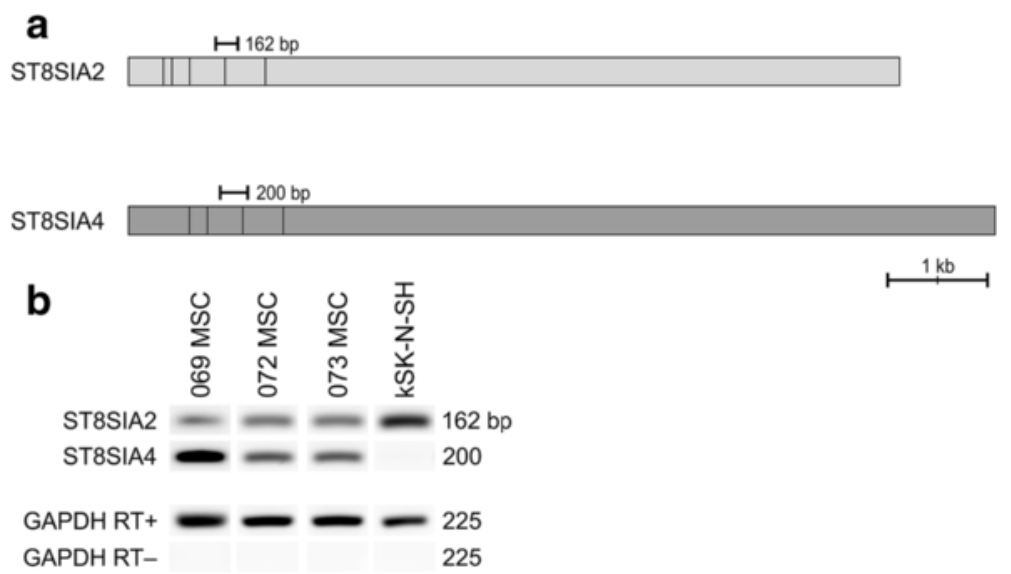

Fig. 3 Polysialyltransferase transcription in hBM-MSCS. NCAM polysialylation is catalyzed by two Golgi resident enzymes, polysialyltransferases ST8SIA2 and ST8SIA4. Qualitative reverse transcription PCR analysis was performed to indicate the presence of polysialyltransferases. a ST8SIA2 gene is located in chromosome $15 q 26$ and consists of six exons, whereas ST8SIA4 gene is located in chromosome $5 q 21$ and is comprised of five exons. b Both polysialyltransferases are transcribed in hBM-MSCs. The polySia-expressing neuroblastoma cell line kSK-N-SH was used as a control. bp base pairs, GAPDH glyceraldehyde 3-phosphate dehydrogenase, MSC mesenchymal stromal cell, RT reverse transcriptase

migration, cytokine response, cell contact-dependent differentiation, and immune response modulation [41-44].

To date, definite information about NCAM and polySia expression in hBM-MSCs has been lacking. Because of the intrinsic heterogeneity of the MSC populations, donor variation, and diversity in culture conditions and analysis methods, biomolecular and cytometric characterization of MSCs from different laboratories is not easy to compare [2]. Furthermore, transcriptomic profiling has become a popular method for characterizing MSCs, but protein and mRNA expression levels do not always correlate. Analysis at the protein level is therefore needed before conclusions about the MSC phenotype can be drawn.

To gain more insight into the characteristics of xenofree hBM-MSCs, we have utilized an established clinicalgrade culture protocol based on human platelet lysate [32]. Platelet lysate has been approved as a safe and effective supplement for MSC cultivation in vitro [45]. Conventional cell culture methods involve many animalderived components; however, they are not desirable for clinical-grade cell production because of the increased risk of cross-contamination and host immune reactions [46]. In addition, xenogeneic additives, like bovine sera, may negatively alter the self-renewal and stemness of hBM-MSCs [47]. Because MSCs are very rare in the bone marrow, isolation and in-vitro expansion of the cells is usually required prior to their use. We analyzed noncryopreserved cells at passage 2 or 3 , which is the time point of choice for most MSC applications because it offers the minimum required number of cells that still hold functional potency $[48,49]$. NCAM and polySia expression is possibly altered during in-vitro culture. However, analysis of the properties of these cells directly from the bone marrow would be challenging because reliable markers for their identification are lacking. Culture of MSCs for at least two passages is commonly used to attain population purity [48].

Our findings regarding NCAM expression in hBMMSCs differ from those reported previously [19-25], and show-in contrast to the generally held view-that the cells in fact do express NCAM. Furthermore, we conducted a more detailed analysis regarding NCAM gene and protein expression. Our data show that all five known NCAM isoforms are transcribed in hBM-MSCs. In particular, the main isoforms are detected at the protein level. NCAM protein is expressed throughout the cell surface in a clustered manner. However, flow cytometric analysis revealed quite broad donor-specific variation in expression levels between the hBM-MSC lines. This is not unexpected even in our relatively homogeneous donor population (healthy females, age 21-31 years), because it has been reported previously that hBM-MSC cultures are heterogeneous mixtures of cells, the properties and potency of which vary greatly between individual donors independent of age or gender [50-52]. In this study, the donor population was not selected based on any specific characteristic, but samples were obtained from the volunteer donors in the order they came in based on the national guidelines for bone marrow donation eligibility. In Finland, as in most other western countries, the majority of bone marrow donors are female [53]. In general, gender has little effect on hBM-MSC features [50, 54, 55].

There are many possible reasons for the conflicting reports about NCAM expression. MSCs are cultured under various conditions (e.g., FBS, human serum, platelet lysate, 

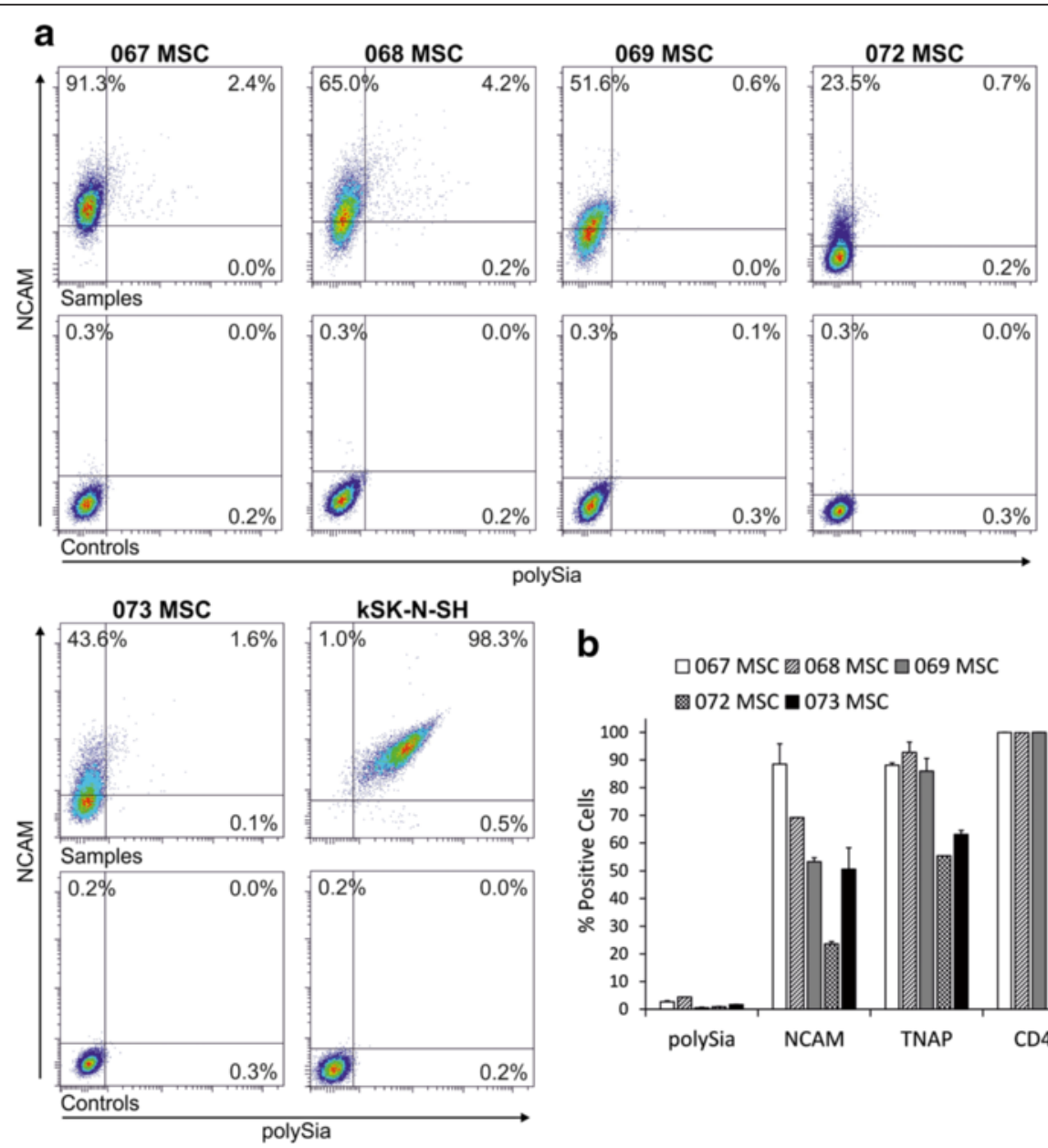

b

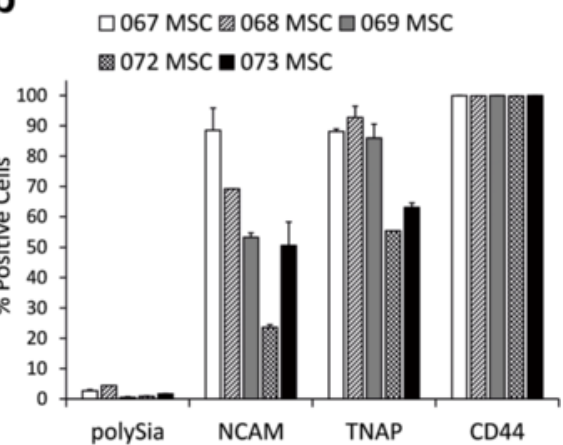

Fig. 4 Flow cytometric analysis of marker expression in hBM-MSCs. Flow cytometric analyses were performed to determine the prevalence of marker expression in hBM-MSC lines. a Plots of NCAM antibody and endosialidase-GFP fusion protein-labeled cells shown in comparison with plots of secondary antibody controls. NCAM was detected in all hBM-MSC lines, but the proportion of positive cells varied. In contrast, the proportion of polySia positive cells was very low. The NCAM and polySia-expressing cell line KSK-N-SH served as a control. b For comparison, hBM-MSC lines were analyzed for the expression of CD44, a common MSC marker, and tissue nonspecific alkaline phosphatase (TNAP), an early osteogenic marker. Standard deviation is indicated. No correlation between marker expressions was detected. MSC mesenchymal stromal cell, NCAM neural cell adhesion molecule, polySia polysialic acid

or serum free) and diverse procedures are employed in their management. Donor-dependent variation may also occur. Expression patterns may be regulated temporally and the cells may be in different stages and passages at the time of analysis, or have undergone replicative senescence. Also, the cellular phenotype may alter between fresh and cryopreserved cells. Technical variation may also be responsible; for example, the variable sensitivity and isoform specificity of anti-NCAM antibodies may give rise to misleadingly low or lacking protein expression. Furthermore, the expression of NCAM mRNA transcripts does not necessarily correlate with the expression of protein on the cell surface.

For comparison, the hBM-MSC lines were also analyzed by flow cytometry for the surface expression of CD44 and TNAP. CD44, a receptor for hyaluronic acid and a common MSC marker, is involved in the contact between stem cells and the niche for stemness maintenance, as well as MSC homing [56, 57]. All five hBMMSC lines expressed high levels of CD44 (>99\%), as expected. TNAP is an early osteogenic marker that is expressed in a stage-specific manner during skeletal development [58]. Furthermore, TNAP deficiency causes bone hypomineralization, abnormalities in brain development, cortical malformations, as well as epileptic seizures [59]. Thus, TNAP and NCAM are developmentally involved in many of the same processes. However, no correlation between TNAP and NCAM expression was observed in the hBM-MSC lines. Also, it has been reported previously that TNAP expression may vary greatly between individual donors $[50,60]$ and our results further support this finding. 


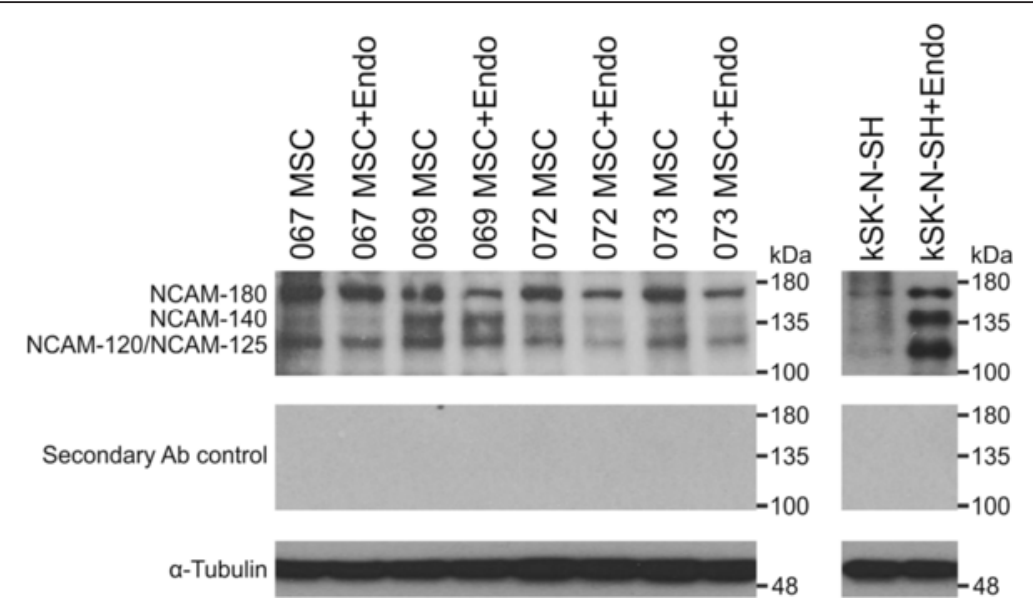

Fig. 5 NCAM protein expression in hBM-MSCs. NCAM is expressed as transmembrane (NCAM-180 and NCAM-140), membrane-anchored (NCAM125 and NCAM-120), or secreted (any isoform) protein. Western blotting was performed to identify which NCAM isoforms are expressed at the protein level in hBM-MSCs. In gel electrophoresis, polySia may affect the mobility of NCAM. Parallel samples were thus treated with endosialidase (+Endo) to remove polySia. NCAM was detected with a mixture of two primary antibodies. All of the main NCAM isoforms-NCAM-180, NCAM140, and NCAM-120 - were detected from the whole cell lysates of hBM-MSCs. The NCAM-120 and NCAM-125 isoforms could not be distinguished from one another. The NCAM-expressing neuroblastoma cell line kSK-N-SH served as a positive control. Secondary antibody control confirmed the specificity of the labeling and a-Tubulin served as a loading control. MSC mesenchymal stromal cell, NCAM neural cell adhesion molecule

To our knowledge, polySia expression in hBM-MSCs has not been reported previously. Our results show that both polysialyltransferases, ST8SIA2 and ST8SIA4, catalyzing polySia synthesis are transcribed in these cells. However, very few cells express polySia on the cell surface. On closer examination it was perceived that polySia is expressed mostly on the cell extensions, in accordance with its natural role as a promoter of cell projection outgrowth and targeting [61]. Difference in polysialyltransferase expression and polysialylation levels is an interesting finding, because traditionally it is thought that expression of polySia correlates with transcription of polysialyltransferases [62, 63]. However, it has been previously shown that other, calcium-dependent, nontranscriptional regulatory pathways also exist [64]. Such nontranscriptional regulation may be due to the spatiotemporal nature of
polySia, requiring specific cues for prompt and selective expression on the cell surface $[65,66]$.

Different cell types express different glycan signatures, a property which has also been utilized to identify and purify stem cells [67]. For example, the glycolipids SSEA-3 and SSEA-4 are amongst the most commonly used markers to identify embryonic stem cells; however, they are not necessary for the maintenance of pluripotency [68]. It is well known that expression of polysialylated NCAM decreases during postnatal development and mostly unpolysialylated NCAM is expressed in adult tissues, where it regulates cell interactions independent of polySia $[69,70]$. In addition, a recent study shows that polysialylation is regulating human pluripotent stem cell differentiation into the three germ layers [63]. In the mesoderm, ST8SIA4 is the principle polysialyltransferase
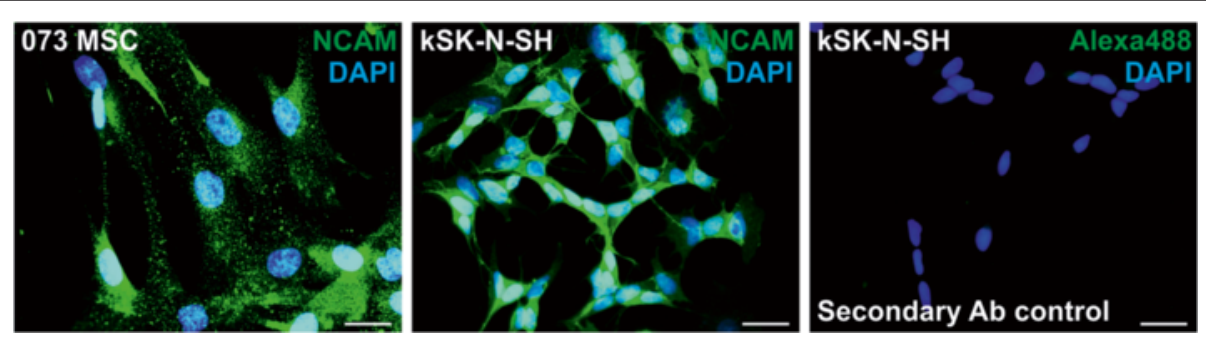

Fig. 6 NCAM localization in hBM-MSCs. Immunofluorescence staining was performed to study NCAM localization. Exemplified fluorescence microscopic photographs of antibody-labeled nonpermeabilized cells. On hBM-MSCs, NCAM is expressed in a clustered manner around the cell surface. In contrast, NCAM expression on KSK-N-SH cells appears to be even and smooth, and concentrating towards cell-cell contact sites. Secondary antibody control confirmed the specificity of the staining. Scale bar $=25 \mu \mathrm{m}$. DAPI 4, 6-diamidino-2-phenylindole, MSC mesenchymal stromal cell, NCAM neural cell adhesion molecule 

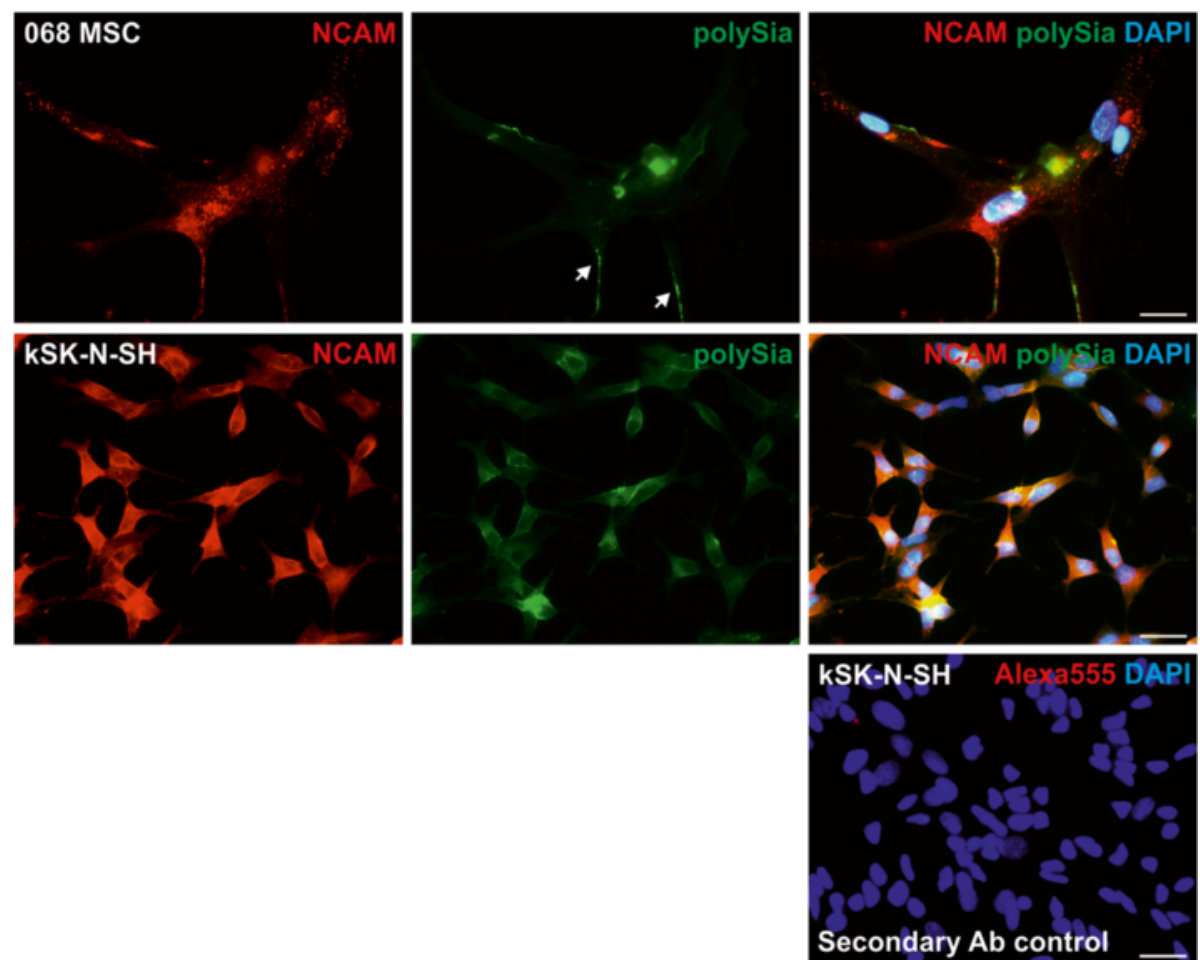

Fig. 7 PolySia localization in hBM-MSCs. Immunofluorescence staining was performed to study polySia localization. Exemplified fluorescence microscopic photographs of antibody and fusion protein-labeled nonpermeabilized cells. Only very little polySia was detected on hBM-MSCs, mostly on cell extensions (white arrows). In contrast, polySia expression on kSK-N-SH cells is fairly strong and smooth throughout the cell surface. Secondary antibody control confirmed the specificity of the staining. Scale bar $=25 \mu \mathrm{m}$. DAPI 4',6-diamidino-2-phenylindole, MSC mesenchymal stromal cell, NCAM neural cell adhesion molecule, polySia polysialic acid

under normal conditions, but this switches to ST8SIA2 when ST8SIA4 activity is eliminated [63]. The observed expression pattern of polysialyltransferases and restricted polysialylation may thus indicate that hBM-MSCs are different from their prenatal pluripotent counterparts. However, our differentiation results evidently demonstrate that these cells still possess multilineage differentiation capacity. Furthermore, the uncovered polySia and NCAM expression may provide novel targets to modify MSC function [71].

\section{Conclusions}

Despite some promising clinical results related to refractory graft-versus-host disease, the biological properties of MSCs remain largely unknown and clinical MSC applications are lacking good markers, which reflect the clinical efficacy of the cells. Generally, NCAM expression is considered to be absent from hBM-MSCs, but the results of our gene and protein expression analysis show that clinical-grade hBM-MSCs do, in fact, express NCAM as well as polySia. Hence, these results underline the need for comprehensive analyses and careful control of methods in the characterization of MSCs. NCAM and polySia represent promising new candidates to influence
MSC interactions. However, their functional and clinical significance needs to be explored in further studies.

\section{Abbreviations}

CAM, cell adhesion molecule; DAPI, 4',6-diamidino-2-phenylindole; FBS, fetal bovine serum; FMO, fluorescence minus one; GFP, green fluorescent protein; hBM-MSC, human bone marrow-derived mesenchymal stromal cell; MSC, mesenchymal stromal cell; NCAM, neural cell adhesion molecule; PBS, phosphate-buffered saline; polySia, polysialic acid; ST8SIA2, ST8 alpha- $N$ acetyl-neuraminide alpha-2,8-sialyltransferase 2; ST8SIA4, ST8 alpha-N-acetylneuraminide alpha-2,8-sialyltransferase 4; TNAP, tissue nonspecific alkaline phosphatase

\section{Acknowledgements}

The authors thank Sirkka Hirschovits-Gerz and Susanna Räsänen (Finnish Red Cross Blood Service) for expert technical assistance. They also thank Dr Antti Rivinoja and Dr Reinhard Schwartz-Albiez (DKFZ (German Cancer Research Center), Translational Immunology, Heidelberg, Germany) for proposing isoform-specific PCR and providing most of the primer sequences. This study was supported by the Integrative Life Science Doctoral Program (ILS) of University of Helsinki, Finska Läkaresällskapet, the Academy of Finland (Grant 138365), and the Magnus Ehrnrooth Foundation.

\section{Authors' contributions}

JF and MIP conceived and supervised the study. MSS, JN, HA, TAL, MIP, and JF designed the experiments. MSS and JN performed the experiments. JN and MK provided ethical approval, cells, and reagents. JF provided tools and reagents. MSS wrote the manuscript. All authors made manuscript revisions. All authors read and approved the manuscript. 


\section{Competing interests}

The authors declare that they have no competing interests.

\section{Author details}

${ }^{1}$ Biochemistry and Biotechnology, Department of Biosciences, University of Helsinki, P.O. Box 56, Fl-00014 Helsinki, Finland. ${ }^{2}$ Cell Therapy Services, Finnish Red Cross Blood Service, Kivihaantie 7, Fl-00310 Helsinki, Finland. ${ }^{3}$ Present Address: Genoscoper Laboratories Oy, P.O. Box 1040, FI-00251 Helsinki, Finland. ${ }^{4}$ Present Address: Department of Bacteriology and Immunology, Medicum, Research Programs Unit, Immunobiology, University of Helsinki, P.O. Box 21, Fl-00014 Helsinki, Finland.

Received: 21 December 2015 Revised: 28 June 2016 Accepted: 21 July 2016 Published online: 15 August 2016

\section{References}

1. Bara JJ, Richards RG, Alini M, Stoddart MJ. Concise review: Bone marrowderived mesenchymal stem cells change phenotype following in vitro culture: implications for basic research and the clinic. Stem Cells. 2014;32:1713-23.

2. Phinney DG. Biochemical heterogeneity of mesenchymal stem cell populations: clues to their therapeutic efficacy. Cell Cycle. 2007;6:2884-9.

3. Walmod PS, Kolkova K, Berezin V, Bock E. Zippers make signals: NCAMmediated molecular interactions and signal transduction. Neurochem Res. 2004;29:2015-35

4. Cunningham BA, Hemperly JJ, Murray BA, Prediger EA, Brackenbury R, Edelman GM. Neural cell adhesion molecule: structure, immunoglobulin-like domains, cell surface modulation, and alternative RNA splicing. Science. 1987:236:799-806.

5. Finne J, Finne U, Deagostini-Bazin H, Goridis C. Occurrence of alpha 2-8 linked polysialosyl units in a neural cell adhesion molecule. Biochem Biophys Res Commun. 1983;112:482-7.

6. Nelson RW, Bates PA, Rutishauser U. Protein determinants for specific polysialylation of the neural cell adhesion molecule. J Biol Chem. 1995;270:17171-9.

7. Rutishauser U. Polysialic acid and the regulation of cell interactions. Curr Opin Cell Biol. 1996:8:679-84

8. Rutishauser U. Polysialic acid in the plasticity of the developing and adult vertebrate nervous system. Nat Rev Neurosci. 2008;9:26-35.

9. Falconer RA, Errington RJ, Shnyder SD, Smith PJ, Patterson LH. Polysialyltransferase: a new target in metastatic cancer. Curr Cancer Drug Targets. 2012;12:925-39.

10. Schnaar RL, Gerardy-Schahn R, Hildebrandt H. Sialic acids in the brain: gangliosides and polysialic acid in nervous system development, stability, disease, and regeneration. Physiol Rev. 2014;94:461-518.

11. Thiery JP, Duband JL, Rutishauser U, Edelman GM. Cell adhesion molecules in early chicken embryogenesis. Proc Natl Acad Sci U S A. 1982;79:6737-41.

12. Rothbard JB, Brackenbury R, Cunningham BA, Edelman GM. Differences in the carbohydrate structures of neural cell-adhesion molecules from adult and embryonic chicken brains. J Biol Chem. 1982;257:11064-9.

13. Pruszak J, Sonntag KC, Aung MH, Sanchez-Pernaute R, Isacson O. Markers and methods for cell sorting of human embryonic stem cell-derived neural cell populations. Stem Cells. 2007:25:2257-68.

14. Hombach-Klonisch S, Panigrahi S, Rashedi I, Seifert A, Alberti E, Pocar P, et al. Adult stem cells and their trans-differentiation potential-perspectives and therapeutic applications. J Mol Med (Berl). 2008;86:1301-14

15. Lackie PM, Zuber C, Roth J. Polysialic acid of the neural cell adhesion molecule (N-CAM) is widely expressed during organogenesis in mesodermal and endodermal derivatives. Differentiation. 1994:57:119-31.

16. Widelitz RB, Jiang TX, Murray BA, Chuong CM. Adhesion molecules in skeletogenesis: II. Neural cell adhesion molecules mediate precartilaginous mesenchymal condensations and enhance chondrogenesis. J Cell Physiol. 1993;156:399-411.

17. Tavella S, Raffo P, Tacchetti C, Cancedda R, Castagnola P. N-CAM and $\mathrm{N}$-cadherin expression during in vitro chondrogenesis. Exp Cell Res. 1994:215:354-62.

18. Fang J, Hall BK. N-CAM is not required for initiation of secondary chondrogenesis: the role of N-CAM in skeletal condensation and differentiation. Int J Dev Biol. 1999;43:335-42.

19. De Ugarte DA, Alfonso Z, Zuk PA, Elbarbary A, Zhu M, Ashiian P, et al. Differential expression of stem cell mobilization-associated molecules on multi-lineage cells from adipose tissue and bone marrow. Immunol Lett. 2003:89:267-70

20. Vogel W, Grünebach F, Messam CA, Kanz L, Brugger W, Bühring HJ. Heterogeneity among human bone marrow-derived mesenchymal stem cells and neural progenitor cells. Haematologica. 2003;88:126-33.

21. Mariotti E, Mirabelli P, Abate G, Schiattarella M, Martinelli P, Fortunato G, et al. Comparative characteristics of mesenchymal stem cells from human bone marrow and placenta: CD10, CD49d, and CD56 make a difference. Stem Cells Dev. 2008:17:1039-41.

22. Lecourt S, Marolleau JP, Fromigué O, Vauchez K, Andriamanalijaona R, Ternaux B, et al. Characterization of distinct mesenchymal-like cell populations from human skeletal muscle in situ and in vitro. Exp. Cell Res. 2010:316:2513-26.

23. Rozemuller H, Prins HJ, Naaijkens B, Staal J, Bühring HJ, Martens AC. Prospective isolation of mesenchymal stem cells from multiple mammalian species using cross-reacting anti-human monoclonal antibodies. Stem Cells Dev. 2010;19:1911-21.

24. Bosch J, Houben AP, Radke TF, Stapelkamp D, Bünemann E, Balan P, et al. Distinct differentiation potential of "MSC" derived from cord blood and umbilical cord: are cord-derived cells true mesenchymal stromal cells? Stem Cells Dev. 2012:21:1977-88.

25. Jaramillo-Ferrada PA, Wolvetang EJ, Cooper-White JJ. Differential mesengenic potential and expression of stem cell-fate modulators in mesenchymal stromal cells from human-term placenta and bone marrow. J Cell Physiol. 2012;227:3234-42.

26. Brooke G, Tong $H$, Levesque JP, Atkinson K. Molecular trafficking mechanisms of multipotent mesenchymal stem cells derived from human bone marrow and placenta. Stem Cells Dev. 2008:17:929-40.

27. Battula VL, Treml S, Bareiss PM, Gieseke F, Roelofs H, de Zwart P, et al. Isolation of functionally distinct mesenchymal stem cell subsets using antibodies against CD56, CD271, and mesenchymal stem cell antigen-1. Haematologica. 2009:94:173-84.

28. Harichandan A, Sivasubramaniyan K, Bühring HJ. Prospective isolation and characterization of human bone marrow-derived MSCs. Adv Biochem Eng Biotechnol. 2013;129:1-17.

29. Wang $X$, Hisha H, Mizokami T, Cui W, Cui Y, Shi A, et al. Mouse mesenchymal stem cells can support human hematopoiesis both in vitro and in vivo: the crucial role of neural cell adhesion molecule. Haematologica. 2010;95:884-91.

30. Yang HJ, Xia YY, Wang L, Liu R, Goh KJ, Ju PJ, et al. A novel role for neural cell adhesion molecule in modulating insulin signaling and adipocyte differentiation of mouse mesenchymal stem cells. J Cell Sci. 2011;124:2552-60.

31. Shi Y, Xia YY, Wang L, Liu R, Khoo KS, Feng ZW. Neural cell adhesion molecule modulates mesenchymal stromal cell migration via activation of MAPKJERK signaling. Exp Cell Res. 2012;318:2257-67.

32. Laitinen A, Oja S, Kilpinen L, Kaartinen T, Möller J, Laitinen S, et al. A robust and reproducible animal serum-free culture method for clinical-grade bone marrow-derived mesenchymal stromal cells. Cytotechnology. 2016;68:891906.

33. Jokilammi A, Ollikka P, Korja M, Jakobsson E, Loimaranta V, Haataja S, et al. Construction of antibody mimics from a noncatalytic enzyme-detection of polysialic acid. J Immunol Methods. 2004;295:149-60.

34. Skog M, Muhonen V, Nystedt J, Narcisi R, Kontturi LS, Urtti A, et al. Xeno-free chondrogenesis of bone marrow mesenchymal stromal cells: towards clinical-grade chondrocyte production. Cytotechnology. 2015;67:905-19.

35. Pelkonen S, Pelkonen J, Finne J. Common cleavage pattern of polysialic acid by bacteriophage endosialidases of different properties and origins. J Virol. 1989;63:4409-16.

36. Ulm C, Saffarzadeh M, Mahavadi P, Müller S, Prem G, Saboor F, et al. Soluble polysialylated NCAM: a novel player of the innate immune system in the lung. Cell Mol Life Sci. 2013;70:3695-708.

37. Dominici M, Le Blanc K, Mueller I, Slaper-Cortenbach I, Marini F, Krause D, et al. Minimal criteria for defining multipotent mesenchymal stromal cells. The International Society for Cellular Therapy position statement. Cytotherapy. 2006:8:315-7

38. Williams EJ, Furness J, Walsh FS, Doherty P. Activation of the FGF receptor underlies neurite outgrowth stimulated by L1, N-CAM, and N-cadherin. Neuron. 1994:13:583-94.

39. Dityatev A, Dityateva G, Schachner M. Synaptic strength as a function of post- versus presynaptic expression of the neural cell adhesion molecule NCAM. Neuron. 2000;26:207-17. 
40. Ditlevsen DK, Berezin V, Bock E. Signalling pathways underlying neural cell adhesion molecule-mediated survival of dopaminergic neurons. Eur J Neurosci. 2007;25:1678-84.

41. Murakami S, Seki T, Rutishauser U, Arai Y. Enzymatic removal of polysialic acid from neural cell adhesion molecule perturbs the migration route of luteinizing hormone-releasing hormone neurons in the developing chick forebrain. J Comp Neurol. 2000;420:171-81.

42. Petridis AK, El-Maarouf A, Rutishauser U. Polysialic acid regulates cell contact-dependent neuronal differentiation of progenitor cells from the subventricular zone. Dev Dyn. 2004;230:675-84.

43. Zhang H, Vutskits L, Calaora V, Durbec P, Kiss JZ. A role for the polysialic acid-neural cell adhesion molecule in PDGF-induced chemotaxis of oligodendrocyte precursor cells. J Cell Sci. 2004;117:93-103.

44. Drake PM, Nathan JK, Stock CM, Chang PV, Muench MO, Nakata D, et al. Polysialic acid, a glycan with highly restricted expression, is found on human and murine leukocytes and modulates immune responses. J Immunol. 2008;181:6850-8.

45. Fekete N, Rojewski MT, Lotfi R, Schrezenmeier H. Essential components for ex vivo proliferation of mesenchymal stromal cells. Tissue Eng Part C. 2014 20:129-39.

46. Verbeek R. Generation of mesenchymal stem cells as a medicinal product in organ transplantation. Curr Opin Organ Transplant. 2013;18:65-70.

47. Nystedt J, Anderson H, Hirvonen T, Impola U, Jaatinen T, Heiskanen A, et al. Human CMP-N-acetylneuraminic acid hydroxylase is a novel stem cell marker linked to stem cell-specific mechanisms. Stem Cells. 2010;28:258-67.

48. von Bahr L, Sundberg B, Lönnies L, Sander B, Karbach H, Hägglund H, et al. Long-term complications, immunologic effects, and role of passage for outcome in mesenchymal stromal cell therapy. Biol Blood Marrow Transplant. 2012;18:557-64.

49. Heathman TR, Rafiq QA, Chan AK, Coopman K, Nienow AW, Kara B, et al. Characterization of human mesenchymal stem cells from multiple donors and the implications for large scale bioprocess development. Biochem Eng J. 2016;108:14-23.

50. Phinney DG, Kopen G, Righter W, Webster S, Tremain N, Prockop DJ. Donor variation in the growth properties and osteogenic potential of human marrow stromal cells. J Cell Biochem. 1999:75:424-36.

51. Scharstuhl A, Schewe B, Benz K, Gaissmaier C, Bühring HJ, Stoop R. Chondrogenic potential of human adult mesenchymal stem cells is independent of age or osteoarthritis etiology. Stem Cells. 2007;25:3244-51.

52. Siddappa $R$, Licht $R$, van Blitterswijk $C$, de Boer J. Donor variation and loss of multipotency during in vitro expansion of human mesenchymal stem cells for bone tissue engineering. J Orthop Res. 2007;25:1029-41.

53. Studts JL, Ruberg JL, McGuffin SA, Roetzer LM. Decisions to register for the National Marrow Donor Program: rational vs emotional appeals. Bone Marrow Transplant. 2010:45:422-8.

54. Jiang Y, Mishima H, Sakai S, Liu YK, Ohyabu Y, Uemura T. Gene expression analysis of major lineage-defining factors in human bone marrow cells: effect of aging, gender, and age-related disorders. J Orthop Res. 2008;26:910-7.

55. Yang HJ, Kim KJ, Kim MK, Lee SJ, Ryu YH, Seo BF, et al. The stem cell potential and multipotency of human adipose tissue-derived stem cells vary by cell donor and are different from those of other types of stem cells. Cells Tissues Organs. 2014;199:373-83.

56. Khaldoyanidi S. Directing stem cell homing. Cell Stem Cell. 2008;2:198-200

57. Wagner W, Wein F, Roderburg C, Saffrich R, Diehlmann A, Eckstein V, et al. Adhesion of human hematopoietic progenitor cells to mesenchymal stromal cells involves CD44. Cells Tissues Organs. 2008;188:160-9.

58. Miyake T, Cameron AM, Hall BK. Stage-specific expression patterns of alkaline phosphatase during development of the first arch skeleton in inbred C57BL/6 mouse embryos. J Anat. 1997;190:239-60.

59. Estève D, Galitzky J, Bouloumié A, Fonta C, Buchet R, Magne D. Multiple functions of MSCA-1/TNAP in adult mesenchymal progenitor/stromal cells. Stem Cells Int. 2016;2016:1815982.

60. Kim YH, Yoon DS, Kim HO, Lee JW. Characterization of different subpopulations from bone marrow-derived mesenchymal stromal cells by alkaline phosphatase expression. Stem Cells Dev. 2012;21:2958-68.

61. El Maarouf A, Rutishauser U. Removal of polysialic acid induces aberrant pathways, synaptic vesicle distribution, and terminal arborization of retinotectal axons. J Comp Neurol. 2003;460:203-11.

62. Angata K, Fukuda M. Polysialyltransferases: major players in polysialic acid synthesis on the neural cell adhesion molecule. Biochimie. 2003;85: 195-206.
63. Berger RP, Sun YH, Kulik M, Lee JK, Nairn AV, Moremen KW, et al. ST8SIA4 dependent polysialylation is part of a developmental program required for germ layer formation from human pluripotent stem cells. Stem Cells. 2016:34:1742-52.

64. Brusés JL, Rutishauser U. Regulation of neural cell adhesion molecule polysialylation: evidence for nontranscriptional control and sensitivity to an intracellular pool of calcium. J Cell Biol. 1998;140:1177-86.

65. Fredette B, Rutishauser U, Landmesser L. Regulation and activitydependence of N-cadherin, NCAM isoforms, and polysialic acid on chick myotubes during development. J Cell Biol. 1993;123:1867-88.

66. Daston MM, Bastmeyer M, Rutishauser U, O'Leary DD. Spatially restricted increase in polysialic acid enhances corticospinal axon branching related to target recognition and innervation. J Neurosci. 1996;16:5488-97.

67. Lanctot PM, Gage FH, Varki AP. The glycans of stem cells. Curr Opin Chem Biol. 2007;11:373-80.

68. Brimble SN, Sherrer ES, Uhl EW, Wang E, Kelly S, Merrill Jr AH, et al. The cell surface glycosphingolipids SSEA-3 and SSEA-4 are not essential for human ESC pluripotency. Stem Cells. 2007;25:54-62.

69. Oltmann-Norden I, Galuska SP, Hildebrandt H, Geyer R, Gerardy-Schahn R, Geyer $\mathrm{H}$, et al. Impact of the polysialyltransferases ST8Siall and ST8SialV on polysialic acid synthesis during postnatal mouse brain development. J Biol Chem. 2008:283:1463-71.

70. Mühlenhoff M, Oltmann-Norden I, Weinhold B, Hildebrandt H, GerardySchahn R. Brain development needs sugar: the role of polysialic acid in controlling NCAM functions. Biol Chem. 2009;390:567-74.

71. Lee YS, Chuong CM. Adhesion molecules in skeletogenesis: I. Transient expression of neural cell adhesion molecules (NCAM) in osteoblasts during endochondral and intramembranous ossification. J Bone Miner Res. 1992;7: 1435-46.

\section{Submit your next manuscript to BioMed Central} and we will help you at every step:

- We accept pre-submission inquiries

- Our selector tool helps you to find the most relevant journal

- We provide round the clock customer support

- Convenient online submission

- Thorough peer review

- Inclusion in PubMed and all major indexing services

- Maximum visibility for your research

Submit your manuscript at www.biomedcentral.com/submit 